\title{
A Fuzzy Logic controller based Multifunctional Dynamic Voltage restorer in Distribution System
}

\author{
M.Divya Vani ${ }^{1}$, Md.Asif ${ }^{2}$, D.Shobha Rani ${ }^{3}$, B.Ramu ${ }^{4}$, P.Harika ${ }^{5}$ \\ 1,2,3,4,5 (Department of EEE, Vardhaman College of Engineering (Autonomous), JNTU Hyderabad, India)
}

\begin{abstract}
Voltage sag is one of major power quality problem and it became severe to industrial customers. Voltage sag can cause miss-operation to several sensitive electronic equipments. This problem can be mitigated with pre-sag voltage injection method using custom power device, Dynamic Voltage Restorer (DVR). In this paper, emergency control in distribution systems is discussed by a flux-charge-model feedback algorithm using Fuzzy Logic Controller in order to improve the transient response and eliminate the steady-state error in DVR response, respectively. A flux-charge-model feedback algorithm is implemented so that the DVR would act as a large virtual inductance in series with the distribution feeder in fault situations. Controlling the DVR as a virtual inductor would also ensure zero real power absorption during the DVR compensation and thus minimize the stress in the DC link. The proposed system is applied to some disturbances in load voltage caused by induction motors starting, and a three-phase short circuit fault. Simulation results show the capability of the DVR to control the emergency conditions of the distribution systems.
\end{abstract}

Keywords - Dynamic voltage restorer (DVR), voltage sag, voltage swell and Fuzzy logic controller.

\section{Introduction}

Voltage sag and voltage swell are two most important power-quality (PQ) problems that encompass almost $80 \%$ of the distribution system PQ problems [1]. According to the IEEE 1959-1995 standard, voltage sag is the decrease of Voltage from 0.1 to 0.9 p.u. in the rms voltage level at system frequency and with the duration of half a cycle to $1 \mathrm{~min}$ [2]. Short circuits, starting large motors, sudden changes of load, and energization of trans-formers are the main causes of voltage sags [3].According to the definition and nature of voltage sag, it can be found that this is a transient phenomenon whose causes are classified as low- or mediumfrequency transient events [2]. In recent years, considering the use of sensitive devices in modern industries, different methods of compensation of voltage sags have been used. One of these methods is using the DVR to im-prove the PQ and compensate the load voltage [6]-[13].

Previous works have been done on different aspects of DVR performance, and different control strategies have been found. These methods mostly depend on the purpose of using DVR. In some methods, the main purpose is to detect and compensate for the voltage sag with minimum DVR active power injection [4], [5]. Also, the in-phase compensation method can be used for sag and swell mitigation [6]. The multiline DVR can be used for eliminating the battery in the DVR structure and control-ling more than one line [7], [14]. Moreover, research has been made on using the DVR in medium level voltage [8]. Harmonic mitigation [9] and control of DVR under frequency variations [10] are also in the area of research. The closed-loop control with load voltage and current feedback is introduced as a simple method to control the DVR in [15].

In all of the aforementioned methods, the source of disturbance is assumed to be on the feeder which is parallel to the DVR feeder. In this paper, a multifunctional control system is proposed in which the DVR protects the load voltage using controllers when the source of disturbance is the parallel feeders. On the other hand, during a downstream fault, the equipment protects the PCC voltage, limits the fault current, and protects itself from large fault current. Although this latest condition has been described in [11] using the flux control method, the DVR proposed there acts like a virtual inductance with a constant value so that it does not receive any active power during limiting the fault current. But in the pro-posed method when the fault current passes through the DVR, it acts like a series variable impedance (unlike [11] where the equivalent impedance was a constant).

The basis of the proposed control strategy in this paper is that when the fault current does not pass through the DVR, an outer feedback loop of the load voltage with an inner feedback loop of the filter capacitor current will be used. Also, a feed forward loop will be used to improve the dynamic response of the load voltage. Moreover, to improve the transient response, the Fuzzy controller and to eliminate the steady-state error, the $\mathrm{P}+$ Resonant controller are used. But in case the fault current passes through the DVR, using the flux control algorithm [11], the series voltage is injected in the opposite direction and, therefore, the DVR acts like a series variable impedance. 


\section{Dvr Components and Its Basic Operational Principle}

\subsection{DVR Components}

A typical DVR-connected distribution system is shown in Fig. 1, where the DVR consists of essentially a series-connected injection transformer, a voltage-source inverter, an inverter output filter, and an energy storage device that is connected to the dc link. Before injecting the inverter output to the system, it must be filtered so that harmonics due to switching function in the inverter are eliminated. It should be noted that when using the DVR in real situations, the injection transformer will be connected in parallel with a bypass switch (Fig. 1). When there is no disturbances in voltage, the injection transformer (hence, the DVR) will be short circuited by this switch to minimize losses and maximize cost effectiveness. Also, this switch can be in the form of two parallel thyristors, as they have high on and off speed [21]. A financial assessment of voltage sag events and use of flexible ac transmission systems (FACTS) devices, such as DVR, to mitigate them is provided in [22]. It is obvious that the flexibility of the DVR output depends on the switching accuracy of the pulse width modulation (PWM) scheme and the control method. The PWM generates sinusoidal signals by comparing a sinusoidal wave with a saw tooth wave and sending appropriate signals to the inverter switches. A further detailed description about this scheme can be found in [23].

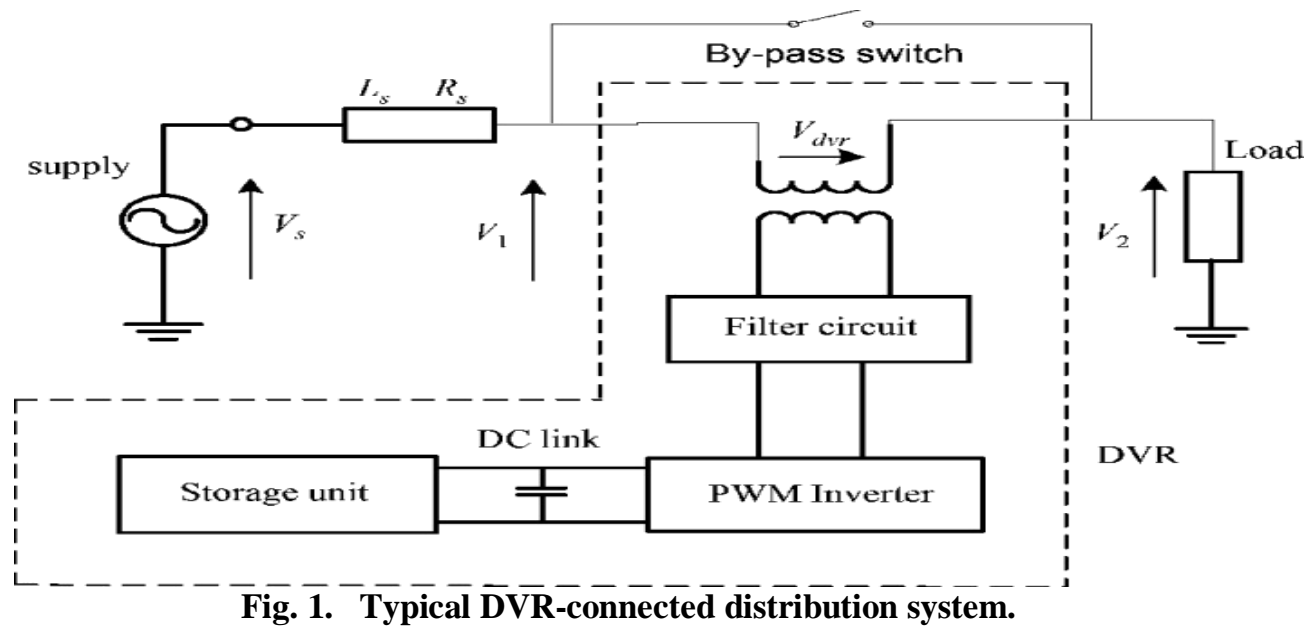

\subsection{Basic Operational Principle of DVR}

The DVR system shown in Fig. 1, controls the load voltage by injecting an appropriate voltage phasor $\Theta_{\mathrm{dvr}}$ in series with the system using the injection series transformer. In most of the sag compensation techniques, it is necessary that during compensation, the DVR injects some active power to the system. There-fore, the capacity of the storage unit can be a limiting factor in compensation, especially during long-term voltage sags.

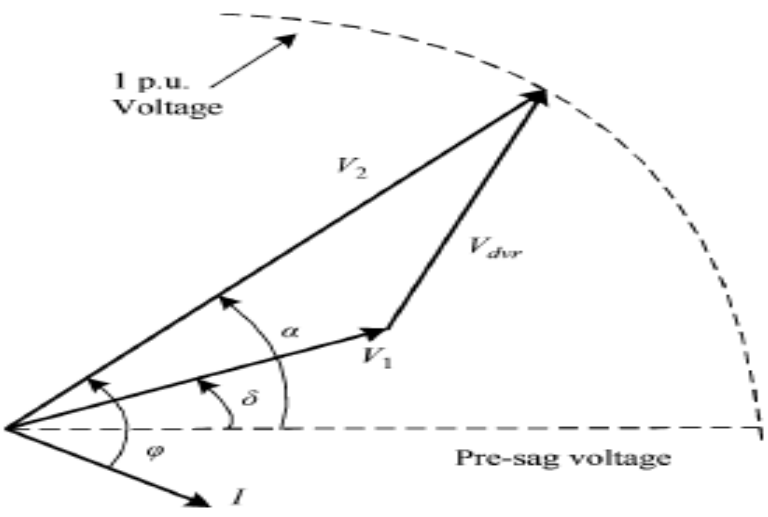

Fig. 2. Phasor diagram of the electrical conditions during a voltage sag.

The phasor diagram in Fig. 2, shows the electrical conditions during voltage sag, where, for clarity, only one phase is shown. Voltages $\mathrm{V}_{1}, \mathrm{~V}_{2}$, and $\mathrm{V}_{\mathrm{dvr}}$ are the source-side voltage, the load-side voltage, and the DVR injected voltage, respectively. Also, the operators I, , and are the load current, the load power factor 
angle, the source phase voltage angle, and the voltage phase advance angle, respectively [24]. It should be noted that in addition to the in-phase injection technique, another technique, namely "the phase advance voltage compensation technique" is also used [24]. One of the advantages of this method over the in-phase method is that less active power should be transferred from the storage unit to the distribution system. This results in compensation for deeper sags or sags with longer durations.

Due to the existence of semiconductor switches in the DVR inverter, this piece of equipment is nonlinear. However, the state equations can be linearized using linearization techniques. The dynamic characteristic of the DVR is influenced by the filter and the load. Although the modeling of the filter (that usually is a simple LC circuit) is easy to do, the load modeling is not as simple because the load can vary from a linear time invariant one to a nonlinear time-variant one. In this paper, the simulations are performed with two types of loads: 1) a constant power load and 2) a motor load.

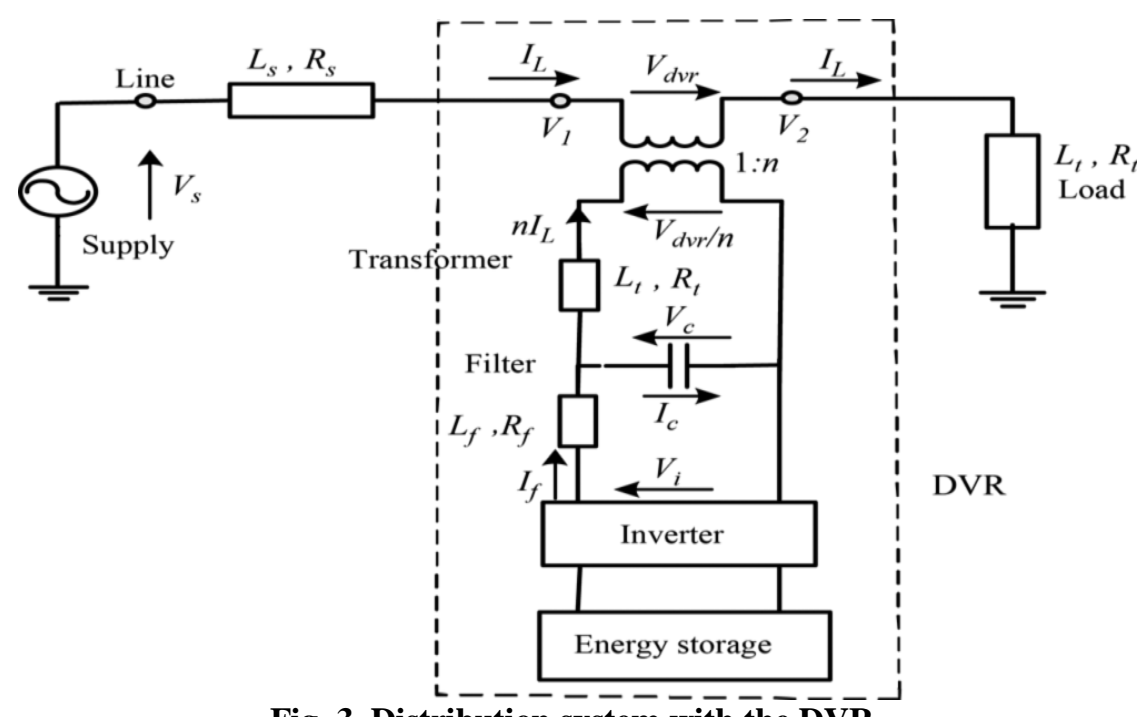

Fig. 3. Distribution system with the $\overline{\mathrm{DVR}} \overline{\text {. }}$

As Fig. 3 shows, the load voltage is regulated by the DVR through injecting $\mathrm{V}_{\mathrm{dvr}}$. For simplicity, the bypass switch shown in Fig. 1 is not presented in this figure. Here, it is assumed that the load has a resistance $R_{1}$ and an inductance $L_{l}$. The DVR harmonic filter has an inductance of $L_{f}$, a resistance of $R_{f}$, and a capacitance of $C_{f}$. Also, the DVR injection transformer has a combined winding resistance of $R_{t}$, a leakage inductance of $L_{t}$, and turns ratio of $1: n$.

\section{Proposed Method For Using The Flux-Charge Model}

In this part, an algorithm is proposed for the DVR to restore the PCC voltage, limit the fault current, and, therefore, protect the DVR components. The flux-charge model here is used in a way so that the DVR acts as a virtual inductance with a variable value in series with the distribution feeder. To do this, the DVR must be controlled in a way to inject a proper voltage having the opposite polarity with respect to usual cases. It should be noted that over current tripping is not possible in this case, unless additional communication between the DVR and the downstream side over current circuit breaker (CB) is available. If it is necessary to operate the over current CB at PCC, communication between the DVR and the PCC breaker might have to be made and this can be easily done by sending a signal to the breaker when the DVR is in the fault-current limiting mode as the DVR is just located after PCC. The proposed DVR control method is illustrated in Fig. 4. It should also be noted that the reference flux (Øref) is derived by integration of the subtraction of the PCC reference voltage (V*PCC) and the DVR load-side voltage. In this control strategy, the control variable used for the outer flux model is the inverter-filtered terminal flux defined as:

$$
\Phi=\int V_{\mathrm{odvr}} \mathrm{dt}
$$




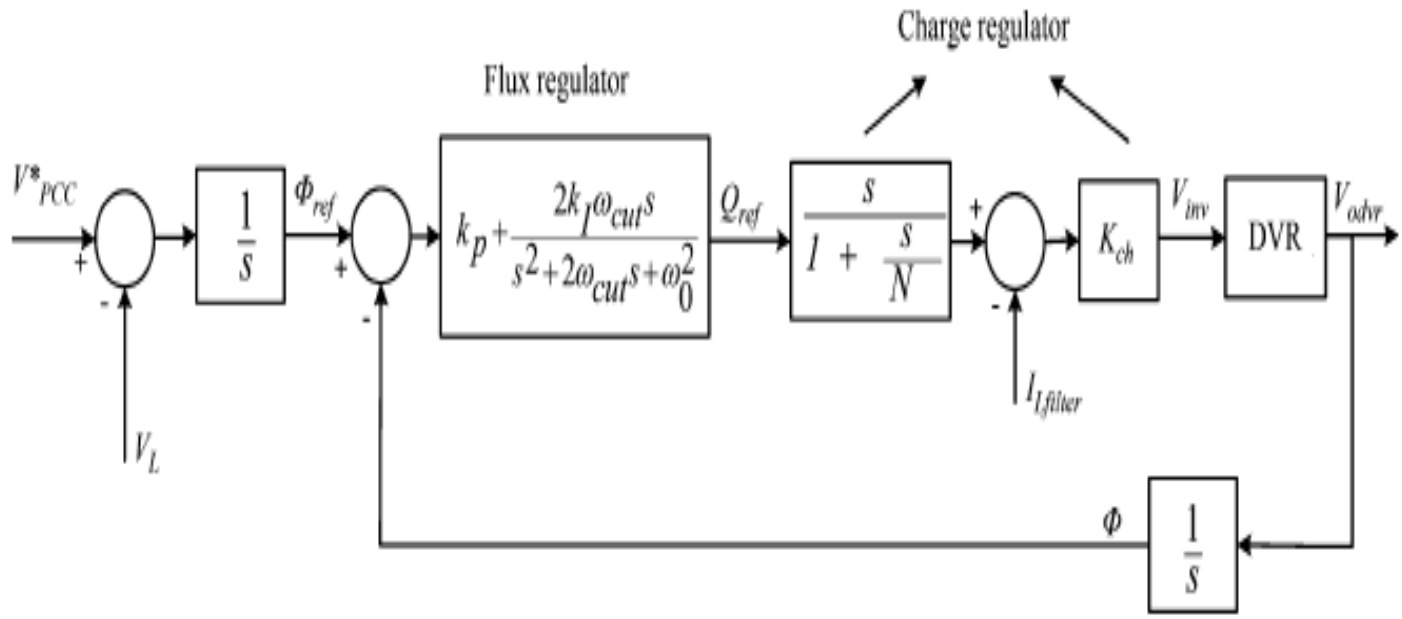

Fig. 4. Proposed method.

Where V0dvr is the filter capacitor voltage of the DVR (at the DVR power converter side of the injection transformer). The flux error is then fed to the flux regulator, which is a P+Resonant controller, with a transfer function given in (6). On the other hand, it can be shown that a single flux-model would not damp out the resonant peak of the LC filter connected to the output of the inverter.

To stabilize the system, an inner charge model is therefore considered. In this loop, the filter inductor charge, which is derived by integration of its current, tracks the reference charge output Qref of the flux regulator. The calculated charge error is then fed to the charge regulator with the transfer function

$$
G_{\text {charge }}(s)=k_{C h} \frac{S}{1+\frac{S}{N}}
$$

Which is actually a practical form of the derivative controller. In this transfer function, the regulator gain is limited to $\mathrm{N}$ at high frequencies to prevent noise amplification.

The derivative term in $\mathrm{S} / 1+\mathrm{S} / \mathrm{N}$ neutralizes the effects of voltage and current integrations at the inputs of the flux-charge model, resulting in the proposed algorithm having the same regulation performance as the multi loop voltage-current feedback control, with the only difference being the presence of an additional lowpass filter in the flux control loop in the form of $1 / 1+\mathrm{S} / \mathrm{N}$. The bandwidth of this low-pass filter is tuned (through varying $\mathrm{N}$ ) with consideration for measurement noise attenuation, DVR LC-filter transient resonance attenuation, and system stability margins.

\section{Simulation Results}

In this part, the proposed DVR topology and control algorithm will be used for emergency control during the voltage sag. The three-phase short circuit and the start of a three-phase large induction motor will be considered as the cause of distortion in the simulations.

\subsection{Under Study Test System}

In this paper, the IEEE standard 13-bus balanced industrial system will be used as the test system. The one-line diagram of this system is shown in Fig. 5.

The test system is modeled in PSCAD/EMTDC software. Control methods of Figs. 5 and 8 were applied to control the DVR, and the voltage, current, flux, and charge errors were included as the figures show. Also, the DVR was modeled by its components (instead of its transfer functions) in the PSCAD/EMTDC software to make more real simulation results. A 12-pulse inverter was used so that each phase could be controlled separately. 


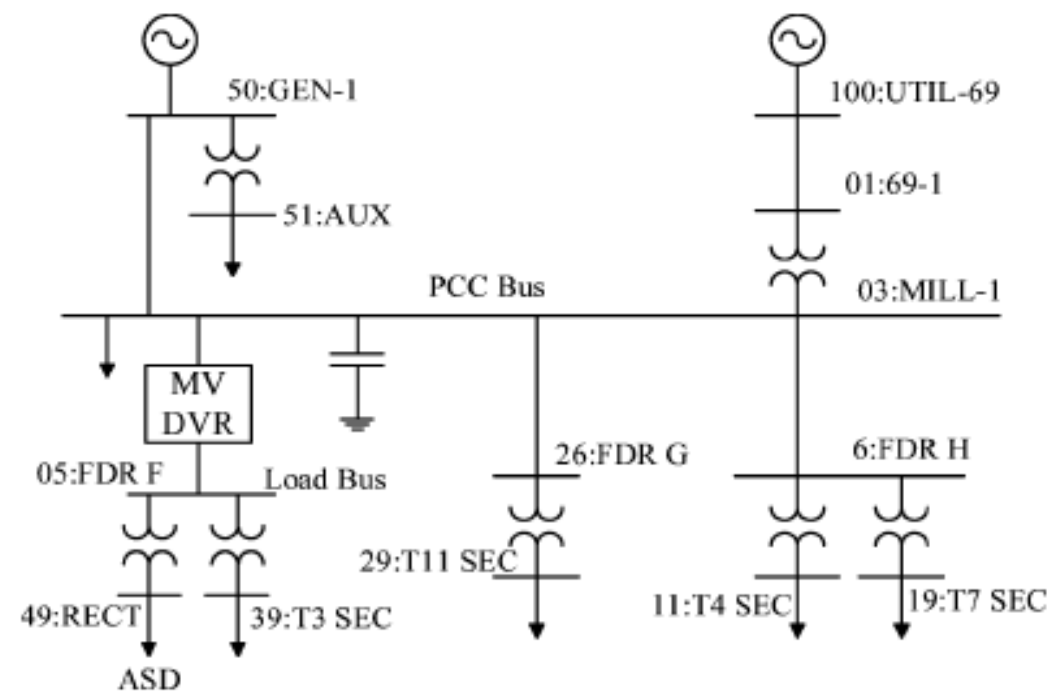

Fig. 5. Under study test system

The plant is fed from a utility supply at $69 \mathrm{kV}$ and the local plant distribution system operates at 13.8 $\mathrm{kV}$. The local (in-plant) generator is represented as a simple Thevenin equivalent. The internal voltage, determined from the converged power-flow solution, is $13.8\left\llcorner-1.52^{\circ} \mathrm{kV}\right.$.

The equivalent impedance is the sub transient impedance which is $0.0366+\mathrm{j}\llcorner 1.3651$. The plant power factor correction capacitors are rated at $6000 \mathrm{kvar}$. As is typically done, leakage and series resistance of the bank are neglected in this study. The detailed description of the system can be found in [25]. In the simulations, the DVR is placed between buses "03:MILL-1" and "05:FDR F."

\subsection{Three-Phase Short Circuit}

In this part, the three-phase short circuit is applied on bus "26:FDR G," and the capability of the DVR in protecting the voltage on bus "05:FDR F" will be studied. The DVR parameters and the control system specifications are provided in Appendices A and B. At $\mathrm{t}=205 \mathrm{~ms}$, the fault is applied at $285 \mathrm{~ms}$, and the breaker works and separates the line between buses "03:MILL-1" and "26:FDR G" from the system. At $\mathrm{t}=305 \mathrm{~ms}$, the fault will be recovered and, finally, at $\mathrm{t}=310 \mathrm{~ms}$, the separated line will be rejoined to the system by the breaker. The simulation results are shown in Fig. 6.
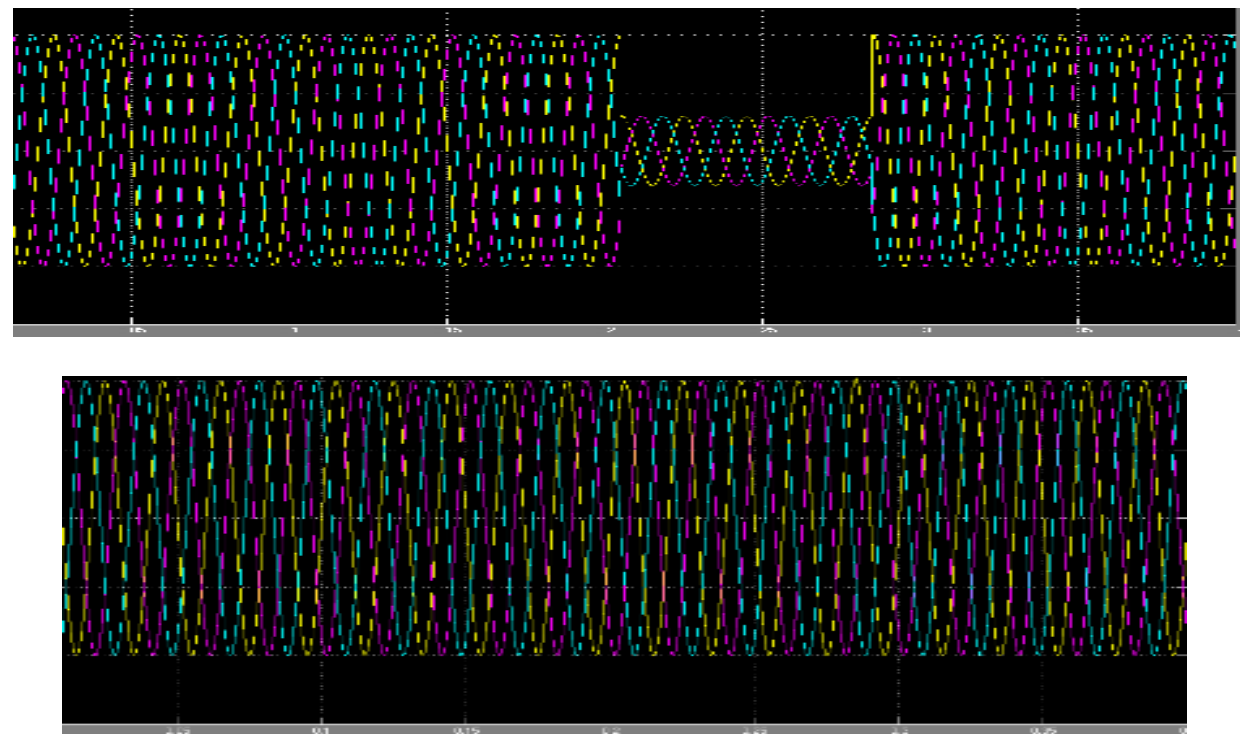


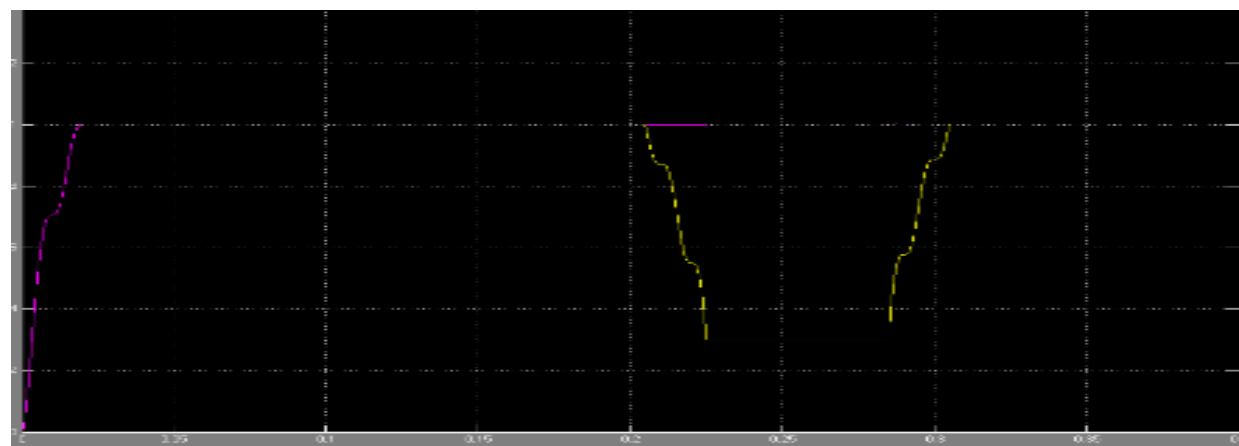

Fig. 6. Three-phase fault compensation by DVR. (a) Three-phase PCC voltages. (b) Three-phase load voltages. (c) RMS voltages of PCC and load.

As can be seen in the figure, the rms voltage of PCC drops to about 0.25 p.u. during the fault. It is obvious that this remaining voltage is due to the impedances in the system. The DVR will start the compensation just after the detection of sag. As can be seen in the enlarged figure, the DVR has restored the voltage to normal form with attenuation of the oscillations at the start of the compensation in less than half a cycle. It is worth noting that the amount and shape of the oscillations depends also on the time of applying the fault. As can be seen in the enlarged figure, the voltage value of phase B is nearly zero; this phase has minimum oscillation when the fault starts.

\subsection{Starting the Induction Motor}

A large induction motor is started on bus "03:MILL-1." The large motor starting current will cause the PCC voltage (bus "03:MILL-1" voltage) to drop. The simulation results in the case of using the DVR are shown in Fig. 7. In this simulation, the motor is started at $\mathrm{t}=405 \mathrm{~ms}$. As can be seen in Fig. 7, at this time, the PCC rms voltage drops to about 0.8 p.u. The motor speed reaches the nominal value in about $1 \mathrm{~s}$.
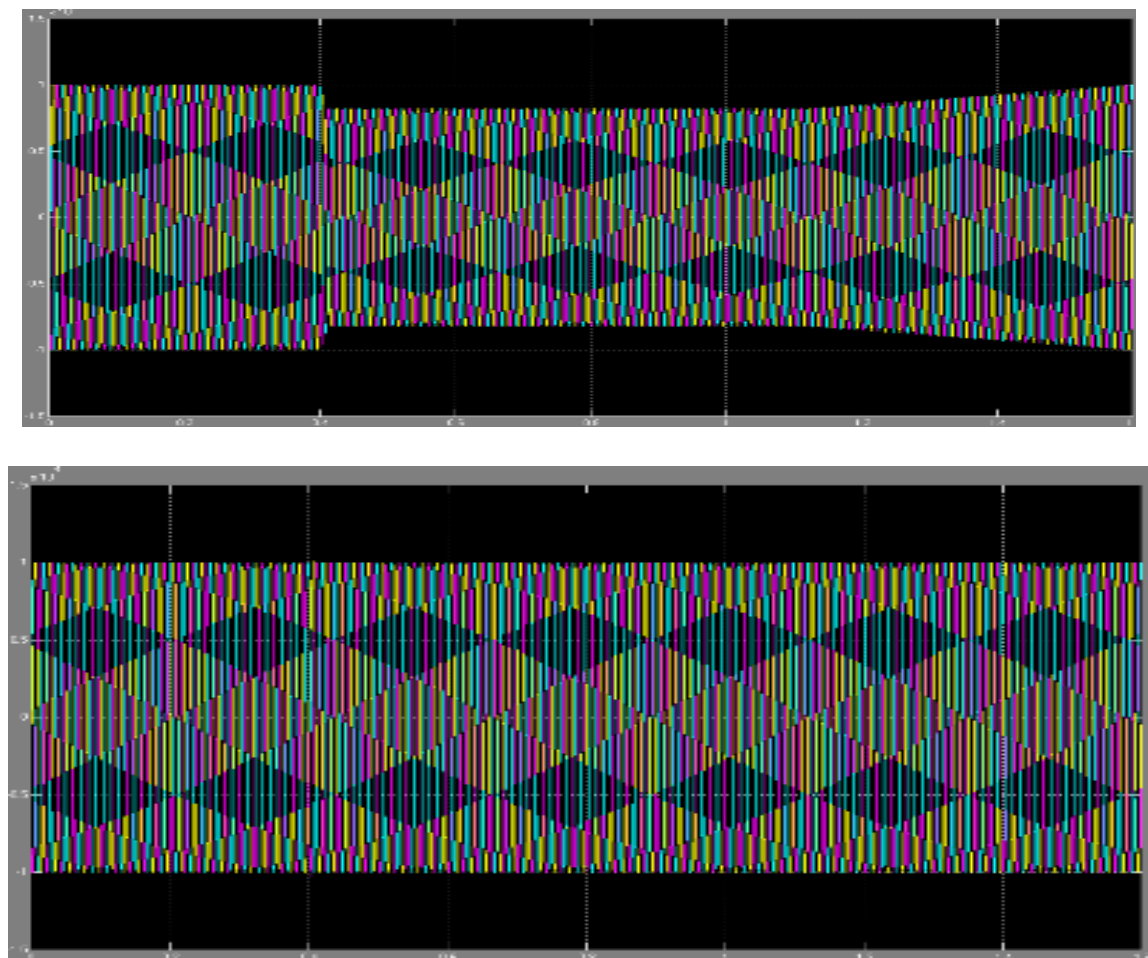


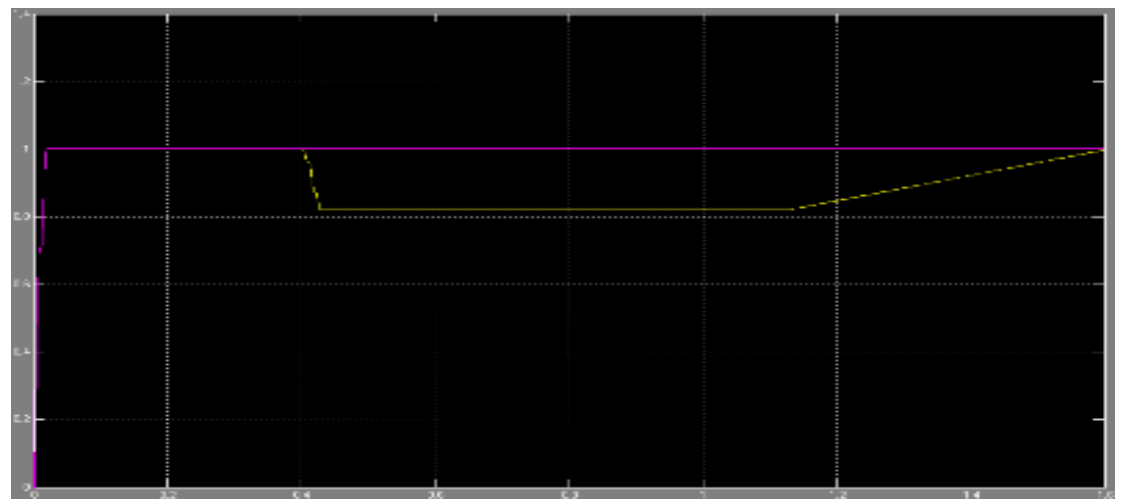

Fig. 7. Starting of an induction motor and the DVR compensation.

(a) Three phase PCC voltages.

(b) Three-phase load voltages.

(c) RMS voltages of PCC and load

During this period, the PCC bus is under voltage sag. From $\mathrm{t}=1.4 \mathrm{~s}$, as the speed approaches nominal, the voltage also approaches the normal condition. However, during all of these events, the DVR keeps the load bus voltage (bus "05:FDR F" voltage) at the normal condition. Also, as can be seen in the enlarged version of Fig. 7, the DVR has succeeded in restoring the load voltage in half a cycle from the instant of the motor starting.

\subsection{Fault Current Limiting}

The last simulation is run for a symmetrical downstream fault, and the capability of the DVR to reduce the fault current and restore the PCC voltage is tested. For this purpose, a three-phase short circuit is applied on bus "05:FDR F". In Fig. 8, the fault current, without the DVR compensation, is shown. For the simulation with DVR compensation, the three-phase fault is applied at $t=205 \mathrm{~ms}$ and then removed after $0.1 \mathrm{~s}$. Also, a breaker will remove the faulted bus from the entire system at $t=300 \mathrm{~ms}$. Fig. 9 shows the DVR operation during the fault. As can be seen, the rms load bus voltage reaches zero during the fault, and as the enlarged figure shows, in about half a cycle, the DVR has succeeded in restoring the PCC voltage wave shape to the normal condition. It should be noted that the amount and shape of the oscillations depend on the time of applying the fault. As Fig. 9 shows, at this time, the voltage value of phase B is nearly zero; this phase has the minimum oscillation when the fault starts. Also, the maximum value of the fault current has been reduced from $40 \mathrm{kA}$ (see Fig. 8) to $5 \mathrm{kA}$ with DVR compensation.

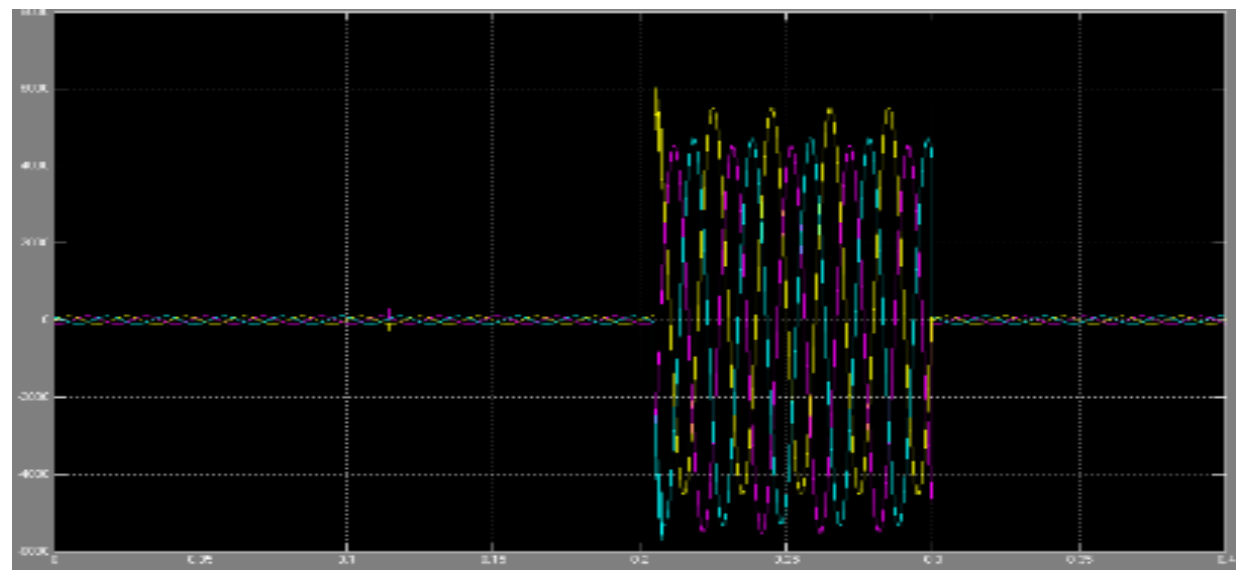

Fig. 8. Current wave shape due to the three-phase short-circuit fault without DVR compensation. 

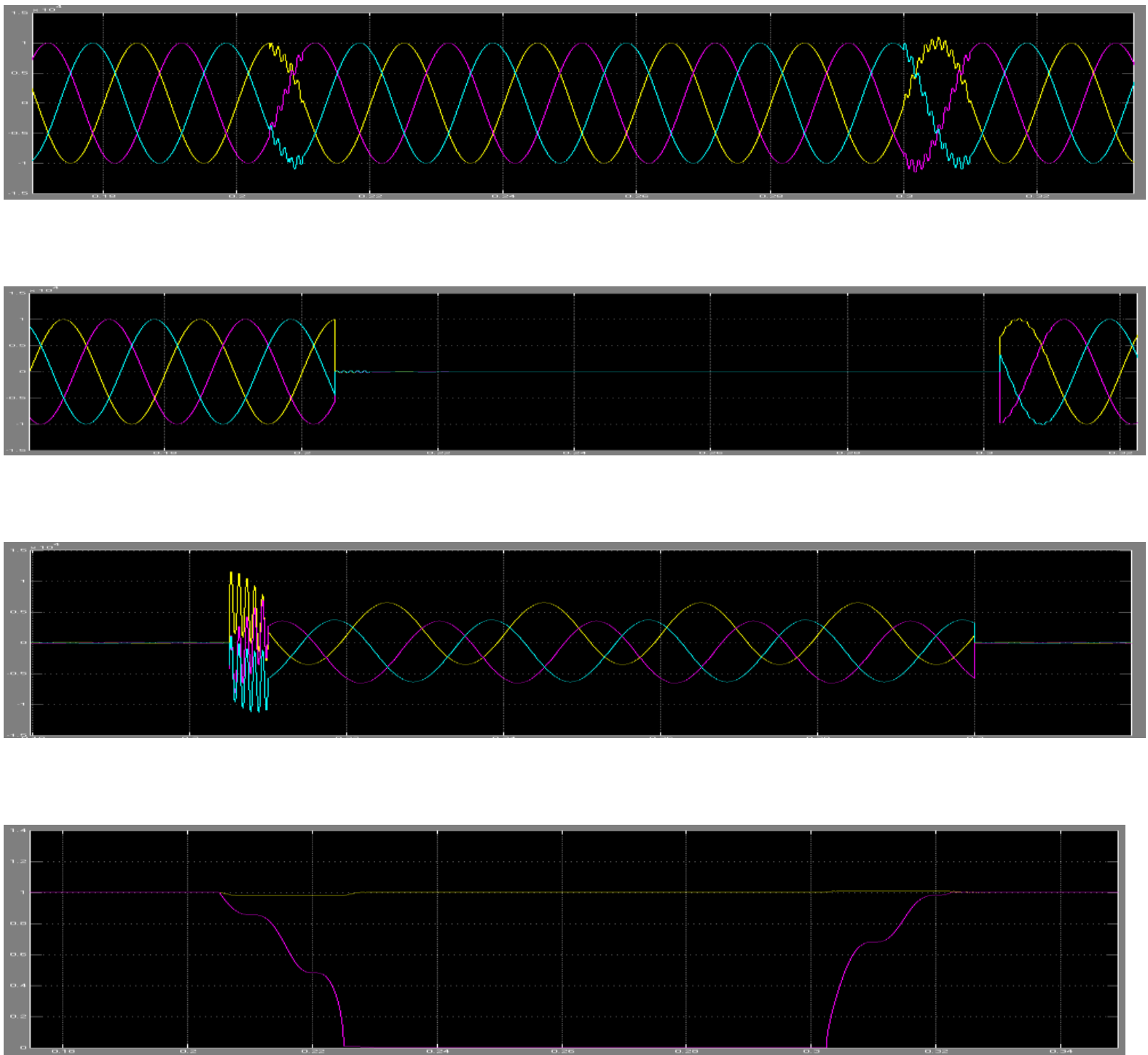

Fig. 9. Fault current limiting by DVR. (a) Three-phase PCC voltages.

(b) Three-phase load voltages. (c) Three- phase currents.

(d) RMS voltages of the PCC and load.

\section{Conclusion}

In this paper, Fuzzy Logic Controlled multifunctional DVR is proposed, and a closed-loop control system is used for its control to improve the damping of the DVR response. Also, for further improving the transient response and eliminating the steady-state error, fuzzy logic controller is used. As the second function of this DVR, using the flux-charge model, the equipment is controlled so that it limits the downstream fault currents and protects the PCC voltage during these faults by acting as variable impedance. The problem of absorbed active power is solved by entering impedance just at the start of this kind of fault in parallel with the dc-link capacitor and the battery being connected in series with a diode so that the power does not enter it. The simulation results verify the effectiveness and capability of the proposed DVR in compensating for the voltage sags caused by short circuits and the large induction motor starting and limiting the downstream fault currents and protecting the PCC voltage.

\section{References}

[1] J. A. Martinez and J. Martin-Arnedo, "Voltage sag studies in distribution networks- part II: Voltage sag assessment," IEEE Trans. Power Del., vol. 21, no. 3, pp. 1679-1688, Jul. 2006

[2] J. A. Martinez and J. M. Arnedo, "Voltage sag studies in distribution networks- part I: System modeling," IEEE Trans. Power Del., vol. 21, no. 3, pp. 338-345, Jul. 2006.

[3] P. Hcine and M. Khronen, "Voltage sag distribution caused by power system faults," IEEE Trans. Power Syst., vol. 18, no. 4, pp. 1367-1373, Nov. 2003.

[4] S. S. Choi, B. H. Li, and D. M. Vilathgamuwa, "Dynamic voltage restoration with minimum energy injection," IEEE Trans. Power Syst., vol. 15, no. 1, pp. 51-57, Feb. 2000. 
[5] C. Fitzer, M. Barnes, and P. Green, “Voltage sag detection technique for a dynamic voltage restore,” IEEE Trans. Ind. Appl., vol. 2, no. 1, pp. 203-212, Jan./Feb. 2004

[6] C. Benachaiba and B. Ferdi, “Voltage quality improvement using DVR,” Electt. Power Qual. Utilisation, Journal, vol. XIV, no. 1, 2008 .

[7] D. M. Vilathgamuwa, H. M. Wijekoon, and S. S. Choi, "A novel technique to compensate voltage sags in multiline distribution system-the interline dynamic voltage restorer," IEEE Trans. Ind. Electron., vol. 53, no. 5, pp. 1603-1611, Oct. 2006.

[8] J. G. Nielsen, M. Newman, H. Nielsen, and F. Blaabjerg, "Control and testing of a dynamic voltage restorer (DVR) at medium voltage level," IEEE Trans. Power Electron., vol. 19, no. 3, pp. 806-813, May 2004.

[9] M. J. Newman, D. G. Holmes, J. G. Nielsen, and F. Blaabjerg, "A dynamic voltage restorer (DVR) with selective harmonic compensation at medium voltage level," IEEE Trans. Ind. Appl., vol. 41, no. 6, pp. 1744-1753, Nov./Dec. 2005.

[10] A. K. Jindal, A. Ghosh, and A. Joshi, "Critical load bus voltage control using DVR under system frequency variation," Elect. Power Syst. Res., vol. 78, no. 2, pp. 255-263, Feb. 2008.

[11] Y. W. Li, D. M. Vilathgamuwa, P. C. Loh, and F. Blaabjerg, "A dualfunctional medium voltage level DVR to limit downstream fault currents," IEEE Trans. Power Electron., vol. 22, no. 4, pp. 1330-1340, Jul. 2007.

[12] P. C. Loh, D. M. Vilathgamuwa, S. K. Tang, and H. L. Long, “Multilevel dynamic voltage restorer,” IEEE Power Electron. Lett., vol. 2 , no. 4 , pp. 125-130, Dec. 2004.

[13] E. Babaei, M. Farhadi, and M. Sabahi, "Compensation of voltage disturbances in distribution systems using single-phase dynamic voltage restorer,” Elect. Power Syst. Res., Jul. 2010.

[14] C. N.-M. Ho and H. S.-H. Chung, "Implementation and performance evaluation of a fast dynamic control scheme for capacitorsupported interline DVR,” IEEE Trans Power Electron., vol. 25, no. 8, pp. 1975-1988, Aug. 2010.

[15] M. Vilathgamuwa, A. A. D. R. Perera, and S. S. Choi, "Performance improvement of the dynamic voltage restorer with closed-loop load voltage and current-mode control," IEEE Trans. Power Electron., vol 17, no. 5, pp. 824-834, Sep. 2002.

[16] Y. W. Li, P. C. Loh, F. Blaabjerg, and D.M.Vilathgamuwa, "Investigation and improvement of transient response of DVR at medium voltage level,” IEEE Trans. Ind. Appl., vol. 43, no. 5, pp. 1309-1319, Sep./Oct. 2007.

[17] H. Kim and S. K. Sul, "Compensation voltage control in dynamic voltage restorers by use of feedforward and state feedback scheme," IEEE Trans. Power Electron., vol. 20, no. 5, pp. 1169-1177, Sep. 2005.

[18] M. I. Marei, E. F. El-Saadany, and M. M. A. Salama, "A new approach to control DVR based on symmetrical components estimation," IEEE Trans. Power Del., vol. 22, no. 4, pp. 2017-2024, Oct. 2007.

[19] Y. W. Li, D. M. Vilathgamuwa, F. Blaabjerg, and P. C. Loh, "A robust control scheme for medium-voltage-level DVR implementation,” IEEE Trans. Ind. Electron., vol. 54, no. 4, pp. 2249-2261, Aug. 2007.

[20] S. A. Saleh, C. R. Moloney, and M. A. Rahman, "Implementation of a dynamic voltage restorer system based on discretewavelet transforms," IEEE Trans. Power Del., vol. 23, no. 4, pp. 2360-2375, Oct. 2008.

[21] H. Awad, J. Svensson, and M. Bollen, “Mitigation of unbalanced voltage dips using static series compensator,” IEEE Trans Power Electron., vol. 19, no. 3, pp. 837-846, May 2004.

[22] J. V. Milanovic and Y. Zhang, "Global minimization of financial losses due to voltage sags with FACTS based devices," IEEE Trans. Power Del., vol. 25, no. 1, pp. 298-306, Jan. 2010.

[23] M. H. Rashid, Power Electronics-Circuits, Devices and Applications, 3rd ed. India: Prentice-Hall of India, Aug. 2006.

[24] M. Vilathgamua, A. A. D. R. Perara, S. S. Choi, and K. J. Tseng, "Control of energy optimized dynamic voltage restorer," in Proc. IEEE IECON Conf., San Jose, CA, 1999, vol. 2, pp. 873-878. 